\title{
Notch is a direct negative regulator of the DNA-damage response
}

\author{
Jelena Vermezovic ${ }^{1}$, Marek Adamowicz ${ }^{1}$, Libero Santarpia ${ }^{2}$, Alessandra Rustighi ${ }^{3,4}$, Mattia Forcato $^{5}$, \\ Caterina Lucano $^{1}$, Lucia Massimiliano ${ }^{6}$, Vincenzo Costanzo ${ }^{1}$, Silvio Bicciato ${ }^{5}$, Giannino Del Sal ${ }^{3,4} \&$ \\ Fabrizio d'Adda di Fagagna ${ }^{1,7}$
}

\begin{abstract}
The DNA-damage response (DDR) ensures genome stability and proper inheritance of genetic information, both of which are essential to survival. It is presently unclear to what extent other signaling pathways modulate DDR function. Here we show that Notch receptor binds and inactivates ATM kinase and that this mechanism is evolutionarily conserved in Caenorhabditis elegans, Xenopus laevis and humans. In C. elegans, the Notch pathway impairs DDR signaling in gonad germ cells. In mammalian cells, activation of human Notch1 leads to reduced ATM signaling in a manner independent of Notch1 transcriptional activity. Notch1 binds directly to the regulatory FATC domain of ATM and inhibits ATM kinase activity. Notch1 and ATM activation are inversely correlated in human breast cancers, and inactivation of ATM by Notch1 contributes to the survival of Notch1-driven leukemia cells upon DNA damage.
\end{abstract}

A DDR signaling cascade is triggered by DNA double-strand breaks (DSBs) and is initiated by the phosphatidylinositol-3-like protein kinase ataxia-telengiectasia mutated (ATM), which phosphorylates multiple substrates at serine or threonine residues preceding a glutamine (pS/TQ). Phosphorylation of the histone H2AX is necessary for signal amplification and recruitment of DDR factors at DNA lesions, and mediator molecules such as p53-binding protein 1 (53BP1) boost the stable accumulation of DDR factors at sites of damage. Signaling is further amplified away from the lesions by the engagement of downstream kinases $\mathrm{CHK} 1$ and $\mathrm{CHK} 2$, which converge onto effector molecules, such as p53 and CDC25, which in turn control cell-cycle arrest, cellular senescence and apoptosis ${ }^{1,2}$.

The components of the DDR apparatus are evolutionarily conserved, and the study of less complex organisms such as C. elegans has proven useful in improving understanding of this signaling pathway $^{3,4}$. The easily accessible germline of C. elegans is thus an excellent system to study DDR activation in a living organism. Most of the DDR factors have orthologs in C. elegans, and some were originally identified in this species ${ }^{4}$.

The Notch signaling pathway is a cell-fate determinant involved in a variety of developmental and physiological processes ${ }^{5,6}$. Notch receptors are released in the nucleus upon interaction with the DeltaSerrate-Lag-2 (DSL) family of ligands, and they regulate expression of different groups of genes depending on the cellular context. Canonical Notch signaling is executed through transcriptional control; however, recent evidence has suggested that other levels of regulation exist that are independent of Notch's transcriptional functions ${ }^{7}$. In addition to directing a number of physiological processes, Notch has oncogenic functions, which have been characterized in a wide range of human cancers $^{8,9}$. However, the mechanisms by which Notch causes cellular transformation are still under intense investigation. Here, we set out to study whether the Notch signaling pathway has an effect on DDR.

\section{RESULTS}

Notch and ATM activity are inversely correlated in C. elegans We previously reported that an anti-pS/TQ antibody specifically detects the phosphorylated substrates of ATM only in the germline cells of the gonad and not in any somatic cell of wild-type worms ${ }^{10}$. We further analyzed pS/TQ signals at the single-cell level in isolated gonads in wild-type worms. Within the anatomical organization of the C. elegans germline, mitotic germ stem cells are morphologically distinct and are spatially separated from those undergoing meiotic differentiation (Supplementary Fig. 1a). Proliferation and stemness of the mitotic compartment is maintained by the distal tip cell at the apical edge of the gonad, which exerts its activity by producing a ligand for the Notch receptor (GLP-1 receptor in C. elegans) expressed on the surface of germ stem cells. We reconfirmed results from our previous report ${ }^{10}$ showing that upon irradiation ATM is robustly activated in all cells of the meiotic compartment, which make up the largest cell population within the gonad. However, we also noticed that ATM activity was impaired in the mitotic compartment, which is composed of a pool of proliferating germ stem cells (Fig. 1a). The lack of ATM activation

\footnotetext{
${ }^{1}$ Istituto Fondazione Italiana per la Ricerca sul Cancro di Oncologia Molecolare, Milan, Italy. ${ }^{2}$ Experimental Therapeutics Unit, Istituto di Ricovero e Cura a Carattere Scientifico Humanitas Clinical and Research Institute, Milan, Italy. ${ }^{3}$ Laboratorio Nazionale del Consorzio Interuniversitario per le Biotecnologie, Trieste, Italy. ${ }^{4}$ Department of Life Sciences, University of Trieste, Trieste, Italy. ${ }^{5}$ Center for Genome Research, University of Modena and Reggio Emilia, Modena, Italy.

${ }^{6}$ European Institute of Oncology, Milan, Italy. ${ }^{7}$ Istituto di Genetica Molecolare, Consiglio Nazionale delle Ricerche, Pavia, Italy. Correspondence should be addressed to F.d'A.d.F. (fabrizio.dadda@ifom.eu).
} 
was not due to reduced ATM expression because ATM transcripts were uniformly and readily detectable by RNA in situ hybridization throughout the entire germline (Supplementary Fig. 1b).

We therefore tested whether Notch-GLP-1 signaling is involved in the control of ATM activation by using $g l p-1$ temperature-sensitive mutant worm strains that show increased or decreased Notch-GLP-1 receptor activity. $g l p-1$ (ar202) is a gain-of-function mutant that, at the restrictive temperature, leads to an increased number of germ cells and ultimately to the formation of an additional ectopic mass of mitotic cells at the proximal end of the germline ${ }^{11}$. When we exposed $g l p-1$ (ar202) mutant worms to the restrictive temperature and then exposed them to irradiation (IR), we detected pS/TQ signal only in the meiotic compartment (Fig. 1b). When we grew $g l p-1$ (ar202) mutants at the restrictive temperature for a longer period, the large tumorous mass of mitotic cells filling the entire gonad was devoid of the pS/TQ signal observed in wild-type worms after exposure to IR (Fig. 1b). When these worms were shifted back to the permissive temperature, cells promptly reacquired their ability to activate ATM (Supplementary Fig. 1c) and displayed a nuclear morphology typical of mitotic cells in wild-type worms.

We next used a $g l p-1(q 224)$ mutant strain bearing a temperaturesensitive loss-of-function mutation in $g l p-1$ (ref. 12). When we exposed $g l p-1$ (q224) worms to IR at the restrictive temperature and stained them for $\mathrm{pS} / \mathrm{TQ}$, we observed full ATM activation throughout the gonad, including a signal at the distal end that was stronger than that observed in the same region in wild-type worms (Fig. 1b). Thus, mitotic germ stem cells of $C$. elegans show reduced ATM activation upon exposure to IR, and reduced Notch-GLP-1 activity results in an increase in ATM signaling, whereas increased Notch-GLP-1 activity leads to reduced ATM activation.

\section{DDR is inactivated in human cells expressing Notch1}

Because the Notch signaling pathway is evolutionarily conserved, we further explored the potential interplay between Notch and ATM in human cells. We expressed in HeLa cells a constitutively active form of human Notch1 that lacks the extracellular domain $(\mathrm{N} 1 \Delta \mathrm{E})$ and is activated in the absence of ligand binding ${ }^{13}$. However, this form of Notch 1 still requires processing by $\gamma$-secretase protease in order for Notch1 to be released from the cell membrane and to enter the nucleus. We exposed N1 $\Delta$ E-expressing cells to IR and stained them for various DDR markers. Strikingly, cells expressing N1 $\Delta$ E showed a dramatic reduction of DDR foci containing phosphorylated ATM (pATM), pS/TQ and 53BP1 as well as individual phosphorylated substrates of ATM kinase (pCHK2T68, pKAP1S824 and pSMC1S966) when compared to control cells (Fig. 1c and Supplementary Fig. 1d-k). However, when we inhibited $\mathrm{N} 1 \Delta \mathrm{E}$ processing by adding a $\gamma$-secretase inhibitor (GSI), DDR was no longer reduced in N1 $\Delta \mathrm{E}$-expressing cells (Fig. 1c). The $\gamma$-H2AX Ser139 signal was not reduced upon N1 $\Delta \mathrm{E}$ expression (Fig. 1c and Supplementary Fig. 1d). Notch1's effect is not the result of altered kinetics of DDR foci formation because formation of foci was impaired up to $24 \mathrm{~h}$ after IR (Supplementary Fig. 11-n).
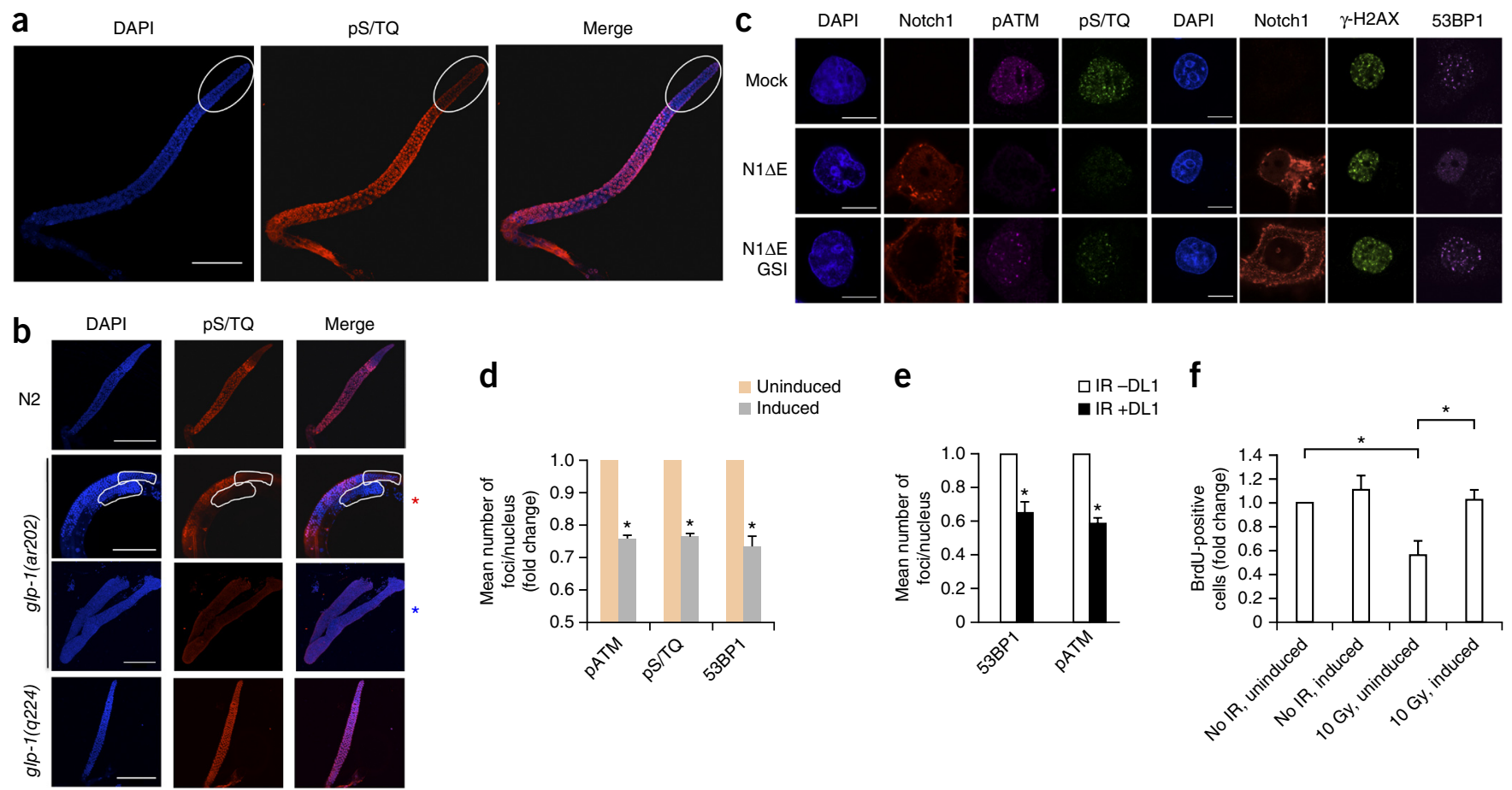

Uninduced

e

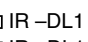

$\mathrm{IR}+\mathrm{DL} 1$

Figure 1 Notch negatively regulates activation of DDR in C. elegans and human cells. (a) Immunostaining for pS/TQ epitopes on dissected gonads of wild-type worms after exposure to IR (180 Gy). The white oval outlines the germ stem cells. Scale bar, $50 \mu \mathrm{m}$. DAPI, 4',6-diamidino-2-phenylindole. (b) Immunostaining for $\mathrm{pS} / \mathrm{TQ}$ epitopes of temperature-sensitive mutants $g / p$-1(ar202) and $g / p$-1(q224). The white lines on the image of $g / p$ 1 (ar202) germline outlines the distal and proximal mitotic compartments. Red asterisk, short exposure (12 h) to the restrictive temperature; blue asterisk, long exposure (from larval stage 1 to adulthood) to the restrictive temperature. Scale bars, $100 \mu \mathrm{m}$. (c) Immunostaining for Notch1 and indicated DDR markers of HeLa cells expressing N1 $\Delta \mathrm{E}$. Cells were treated with GSI and exposed to IR (2 Gy). Scale bars, $10 \mu \mathrm{m}$. (d) Quantification of immunostaining, showing mean number of foci per nucleus, for the indicated DDR markers, in MCF10a cells induced with EGTA and exposed to IR (0.5 Gy). Values are normalized to those of uninduced cells. ${ }^{*} P \leq 0.05$. (e) Quantification of immunostaining, showing mean number of foci per nucleus, for 53BP1 and PATM, in MCF10a cells cocultured with OP9 or OP9-DL1 cells. Values are normalized to those for IR-treated cells with no DL1. ${ }^{*} P \leq 0.005$. (f) Quantification of BrdU-positive MCF10a cells after induction of Notch1 by EGTA and exposure to IR (10 Gy). Values are normalized to those for uninduced cells with no IR. ${ }^{*} P \leq 0.001$. Throughout figure, error bars represent s.e.m. ( $n=3$ independent experiments), and all $P$ values were calculated by two-tailed Student's $t$ test. 


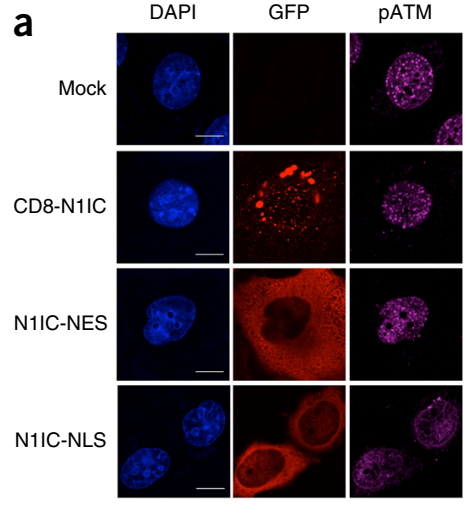

Figure 2 Nuclear Notch1 inactivates DDR in a transcriptionindependent manner. (a) Immunostaining for pATM of irradiated (2 Gy) HeLa cells expressing CD8-N1IC-GFP (cell-membrane bound), GFP-N1IC-NES (predominantly cytosolic) or GFP-N1IC-NLS (predominantly nuclear).

(b) Percentages of cells positive for PATM nuclear foci. NS, $P>0.05$; $P \leq 0.001$. (c) Top, immunoblot showing that the nuclear form of Notch1 (N1IC-NLS) reduces pATM signal. Bottom, blot for GFP, showing that cells express equal levels of all indicated Notch 1 fusion mutants. $\alpha$-tubulin, loading control. (d) Immunostaining for pATM of irradiated (2 Gy) HeLa cells expressing either wild-type, full-length Notch1 (WT) or Notch1 deletion mutant lacking the transactivation domain (Notch1 $\Delta$ TAD). (e) Percentages of cells positive for pATM nuclear foci. ${ }^{*} P \leq 0.001$. (f) Immunostaining for pATM and total ATM of irradiated (2 Gy) HeLa cells expressing N1 $\Delta \mathrm{E}$. (g) Immunoblot with antibody against total ATM, showing equal levels of total ATM in cells expressing either GFP or N1IC-GFP. Samples are from HeLa cells expressing GFP alone or N1IC-GFP, sorted for GFP expression, and exposed to IR (5 Gy). Vinculin is a loading control. Throughout figure, error bars represent s.e.m. ( $n=3$ independent experiments), and all $P$ values were calculated by two-tailed Student's $t$ test. Original immunoblot images are shown in Supplementary Data Set 1 . Scale bars, $10 \mu \mathrm{m}$.

Next, we used nontransformed MCF10a breast epithelial cells, in which endogenous Notch1 activation can be triggered by calcium-ion chelation by EGTA ${ }^{14}$. Automated quantification of the number of DDR foci (Fig. 1d) as well as of the intensity of the DDR signal per nucleus (Supplementary Fig. 1o) demonstrated a significant $(P \leq 0.05)$ reduction in both parameters in irradiated cells with activated Notch1 compared to control cells.

To further support these observations, we used a previously described ${ }^{15}$ physiological system to activate Notch1 receptor, in which MCF10a cells are cultured together with OP9 mouse stromal cells expressing the Delta-like 1 ligand (DL1) for Notch1 receptor. A short incubation time of $3 \mathrm{~h}$ was sufficient to induce detectable levels of Notch 1 in the nuclei of irradiated MCF10a cells cocultured
C
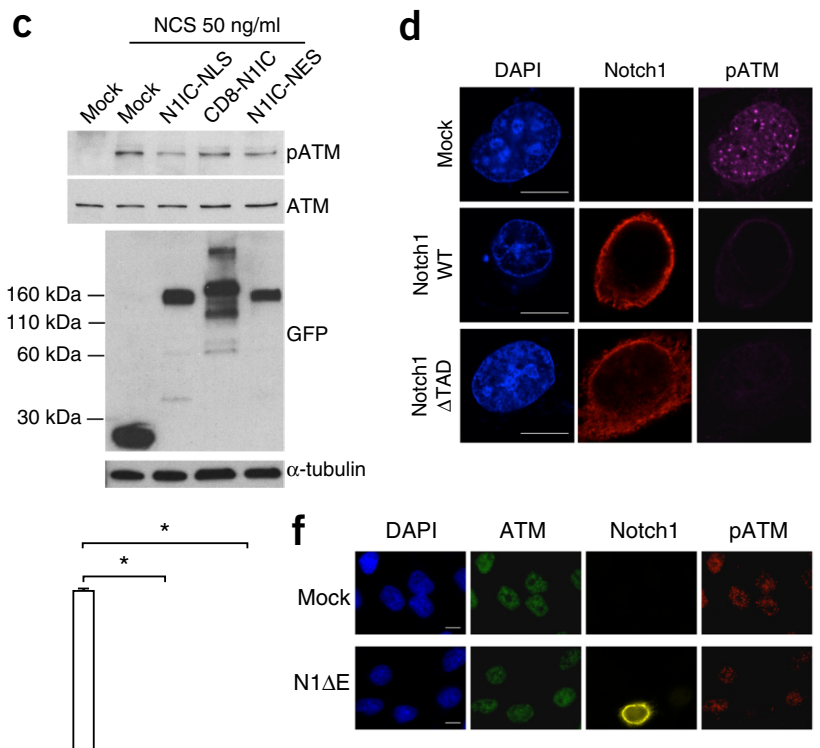

g

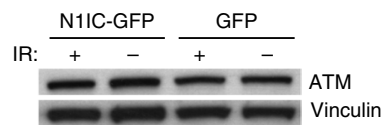

with OP9 cells expressing DL1 ligand (Supplementary Fig. 1p), and it resulted in substantially reduced numbers of pATM and 53BP1 foci (Fig. 1e).

We also analyzed HeLa cells transfected with a viral vector expressing intracellular Notch1 (N1IC) and GFP by fluorescence-activated cell sorting (FACS). Transduced cells expressing moderate levels of N1IC, as reflected by the moderate expression levels of the Notch1 transcriptional target Hes1 (Supplementary Fig. 1q), showed reduced activation of DDR markers pATM, pSMC1, PCHK2 and pKAP1 upon $\mathrm{IR}$, as compared to empty vector-infected cells, whereas $\gamma-\mathrm{H} 2 \mathrm{AX}$ and total DDR protein levels, including 53BP1, remained unaffected (Supplementary Fig. 1r,s). Upon exposure to higher doses of IR (20 Gy), Notch1-expressing cells showed reduced activation of apoptotic
Figure 3 Notch1 and ATM form a protein complex. (a) Immunoblot analysis of an IP with an anti-Flag antibody on lysates of HEK293T cells expressing N1 $\Delta$ E-Flag or an unrelated Flag-tagged protein (control-Flag); input, $5 \%$ of total lysate. (b) Immunoblot analysis of an IP with anti-ATM on lysates of HEK293T cells expressing N1 $\Delta$ E-Flag; input, $5 \%$ of total lysate. (c) Immunoblot analysis of an IP with anti-ATM or the nonrelated antibody (NRA) anti-lgG in MOLT-4 cells; input, $1 \%$ of total lysate. (d) Immunoblot analysis of an IP with anti-N1IC (Val1744) or the NRA anti-myc on CUTLL1 cells; input, $1 \%$ of total lysate. (e) Proximity ligation assay (Duolink). Samples are CUTLL1 cells treated with DMSO or GSI. Notch1 and ATM proteins were labeled fluorescently according to the proximity ligation assay. Scale bars, $10 \mu \mathrm{m}$. (f) Quantification of the mean number of dots per nucleus (Notch1-ATM complexes) normalized to the value of DMSO-treated cells. Error bars, s.e.m. $(n=3)$ independent experiments; ${ }^{*} P \leq 0.001$ by two-tailed Student's $t$ test. Original immunoblot images are shown in Supplementary Data Set 1.

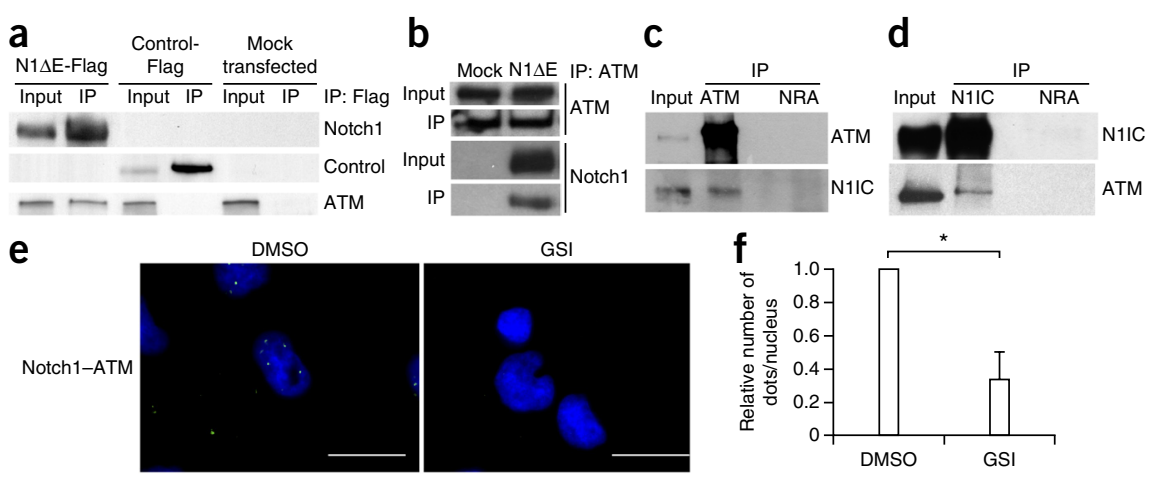


Figure 4 Notch1 binds directly to the FATC domain of ATM and inactivates its kinase activity. (a) Immunoblot analysis of GST pulldown from lysates of HEK293T cells expressing $\mathrm{N} 1 \Delta \mathrm{E}$, incubated with GST-ATM sequential fragments spanning the entire ATM protein (1-12 as indicated) or GST alone; input, $5 \%$ of the lysate used for each reaction. (b) Immunoblot analysis of the in vitro GST-pulldown assay of GST-ATM sequential fragments (1-12) and recombinant purified Flag-tagged human N1IC; input, 100 ng. (c) Immunoblot analysis of in vitro kinase assay in $X$. laevis egg extracts. Double-stranded DNA alone or in the presence of purified, recombinant human Notch 1 was added to the extracts as indicated. $\alpha$-tubulin, loading control. (d) Immunoblot analysis of the in vitro kinase assay with immunopurified human ATM and increasing concentrations $(5,10$ and $20 \mathrm{nM})$ of recombinant N1IC. Double-stranded DNA was added as indicated to elicit phosphorylation of recombinant GST-p53 Ser15 by ATM. Original immunoblot images are shown in Supplementary Data Set 1.

pathways, as measured by cleaved caspase-3 (Supplementary Fig. 1t). We also transduced MCF10a cells with the same viral vector expressing N1IC and confirmed that pATM signal was reduced in the presence of N1IC in this system as well (Supplementary Fig. 1u,v).

Last, we examined the G1-S checkpoint in MCF10a cells upon Notch1 activation after calcium-ion chelation and exposure to IR. Although uninduced cells exposed to IR showed efficient G1-S-checkpoint arrest (reflected as a reduced number of brodmodeoxyuridine (BrdU)-incorporating cells), Notch1-activated cells did not arrest (Fig. 1f), thus indicating that Notch1 activation leads to DNA damage-checkpoint impairment.

\section{Notch1 does not affect DDR activation through transcription} To establish the compartment in which Notch1 exerts its DDR-inhibitory functions, we expressed N1IC fused to a nuclear-export signal (N1IC-NES), to a nuclear-localization sequence (N1IC-NLS) or to an extracellular portion of the CD8 transmembrane receptor that mediates cell-membrane retention (CD8-N1IC) ${ }^{16}$. Staining for DDR markers after IR treatment revealed that only the nuclear form (N1IC-NLS) impaired DDR signaling (Fig. 2a,b and Supplementary Fig. 2a-f). Furthermore, FACS sorting of HeLa cells expressing N1IC fusion proteins and immunoblot analyses of homogenous populations of cells

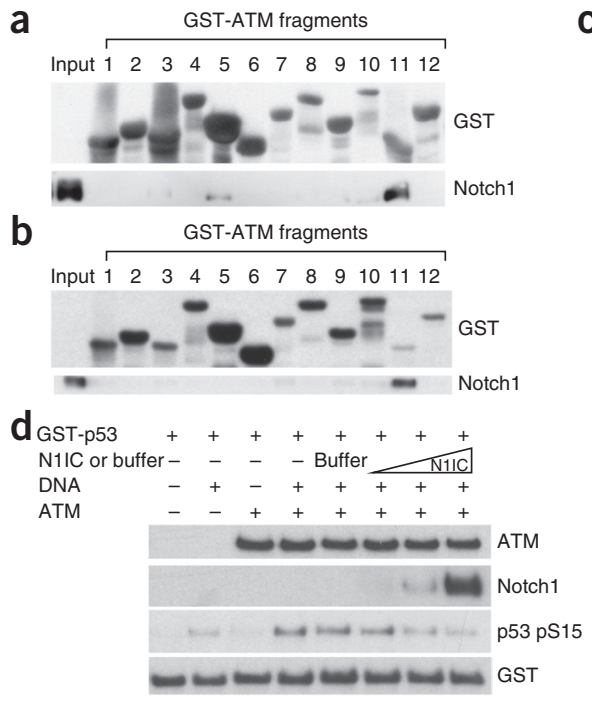

C



expressing Notch1 confirmed that the nuclear form of Notch1 reduces the intensity of pATM to $40 \%$ of that of mock-transfected cells (Fig. 2c and Supplementary Fig. 2g). This result is consistent with the extent of reduction in PATM activation observed by immunofluorescence in the same system (Fig. 2a,b).

Because Notch1 acts as a transcriptional activator of diverse target genes ${ }^{17}$, we examined DDR signaling in cells expressing either fulllength Notch1 or an allele lacking the transcriptional activation domain $(\text { Notch } 1 \triangle T A D)^{18}$. Although the Notch $1 \triangle T A D$ mutant is transcriptionally inactive compared to wild-type Notch1, as shown by impaired induction of the Notch1-target gene HES1 (Supplementary Fig. 2h), we observed similar levels of pATM inactivation (Fig. 2c,d) in cells expressing either full-length Notch1 or Notch1 $\triangle \mathrm{TAD}$, results indicating that DDR inactivation is independent of Notch1 transcriptional activity. Consistently with this observation, total ATM levels, as detected by immunofluorescence and immunoblotting, remained unaffected in cells expressing Notch1 (Fig. 2f,g and Supplementary Fig. 2i).

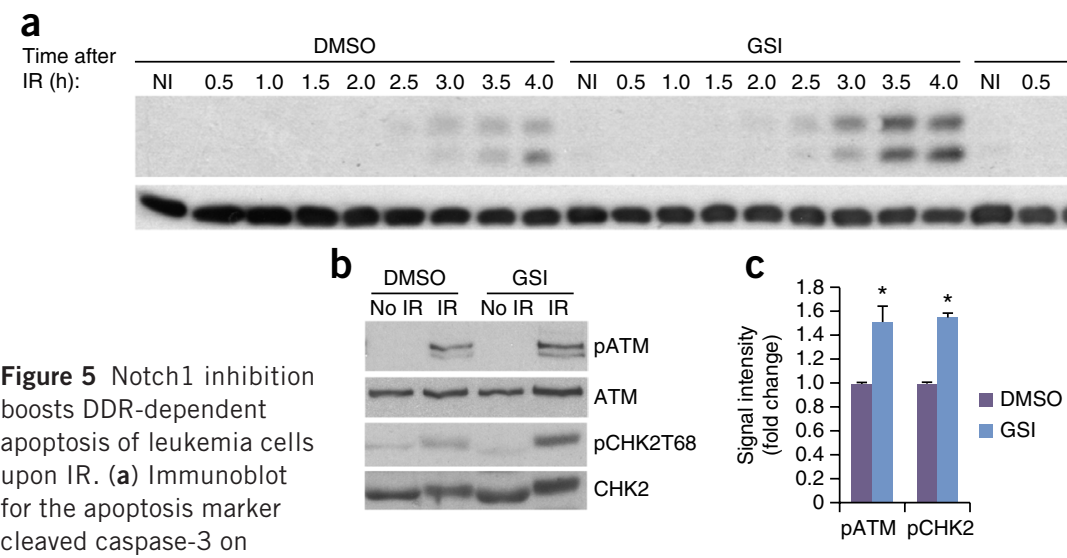

cleaved caspase-3 on

(3 Gy). Cells were collected at indicated time points after exposure to IR or were not irradiated

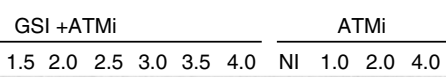

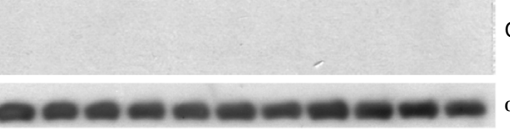

Cleaved caspase-3

$\alpha$-tubulin

(NI). (b) Immunoblot for pATM and pCHK2T68 on lysates of TALL-1 cells treated with GSI and exposed to IR (5 Gy). Cells were collected $1 \mathrm{~h}$ after IR.

(c) Quantification of pATM and pCHK2 Thr68 signals shown in $\mathbf{b}$. The signal intensities of pATM and pCHK2T68 are normalized to the signal intensities of their respective total proteins and to the values of DMSO-treated cells. Error bars, s.e.m. ( $n=3$ independent experiments); $P \leq 0.05$ by twotailed Student's $t$ test. (d) Survival curve of TALL-1 cells upon treatment with GSI and IR and in the presence of ATM inhibitor, as indicated. The time point $-3 \mathrm{~h}$ indicates pretreatment with inhibitors alone; at $0 \mathrm{~h}$, cells were exposed to IR (3 Gy). (e) Survival curve of TALL-1 cells upon treatment with inhibitors (GSI, ATMi, and GSI and ATMi), in the absence of IR. In $\mathbf{d}$ and e, all values are normalized to those before treatments; error bars, s.e.m. ( $n=3$ independent experiments). Original immunoblot images are shown in Supplementary Data Set 1. 
Figure 6 Activated Notch1 inversely correlates with the activation of ATM in breast cancer in vivo. (a) Immunohistochemistry (IHC) of parallel sections of two breast cancer samples: sample 1 with low nuclear Notch1 and high levels of pATM and sample 2 with high nuclear Notch 1 and low levels of pATM. Scale bars, $50 \mu \mathrm{m}$. (b) Scatter plot of quantification of IHC staining for Notch 1 and PATM, scored from no staining (score 0 ) to the highest level of staining (score 3), demonstrating an inverse correlation between the two markers. $P<0.00001$; Spearman $r=-0,4405$ ( $n=89$ tissue samples). (c) Scatter plots of quantification of IHC staining for Notch1 and pATM in a homogeneous cohort of patients showing high levels (score 2 and 3 ) of expression of total ATM. $P<0.001021$ and Spearman $r=-0,5695$ (total ATM score 2); $P<0.009343$ and Spearman $r=-0,4457$ (total ATM score 3) ( $n=89$ tissue samples). (d) Enrichment profile of genes of the ATM-activity signature in tumor samples classified as high NDT signature versus low NDT signature. NDT signature is presented on the bottom colored bar. The color gradient goes from red (samples with high NDT signature) to blue (samples with low NDT signature). The black vertical bars indicate the positions of single components of the ATM-activity signature in NDT samples. The green line shows the cumulative score of the enrichment of the examined ATM-activity signature. A negative enrichment score indicates negative correlation between the signatures.

\section{Notch1 and ATM form a protein complex}

Because ATM inactivation by Notch1 does not occur through transcriptional modulation, and Notch1 function requires nuclear localization, we considered the possibility that Notch1 may interact with ATM in a protein complex. To test this, we coexpressed Flag-tagged ATM and Myc-tagged N1 $\Delta \mathrm{E}$ in human cells and performed an immunoprecipitation (IP) with an anti-Flag antibody. We observed that ATM immunocomplexes contain Notch1 (Supplementary Fig. 3a). In addition, we expressed Flag-tagged $\mathrm{N} 1 \Delta \mathrm{E}$ and found endogenous ATM in a complex with it (Fig. 3a). Importantly, in the same cells expressing Flag-tagged $\mathrm{N} 1 \Delta \mathrm{E}$, a reverse IP for endogenous ATM showed specific interaction with $\mathrm{N} 1 \Delta \mathrm{E}$ (Fig. 3b). The interaction between Notch1 and ATM is not mediated by DNA, because the complex was maintained in the presence of ethidium bromide or after DNase treatment (Supplementary Fig. 3b). We next asked whether IR affects ATM binding to Notch1 by performing reciprocal IPs for ATM or Flag-tagged N1 $\Delta \mathrm{E}$ in cells exposed to IR and found that formation of this protein complex is independent of DNA damage (Supplementary Fig. 3c,d). We additionally noted that levels of the ATM-binding partner NBS1 were not affected by the expression of N1 $\Delta$ E (Supplementary Fig. 3e).

To extend our analyses of the interaction of endogenous ATM and Notch1 proteins, we used two human T-cell acute lymphoblastic leukemia (T-ALL) cell lines, MOLT-4 and CUTLL1, which both express high levels of endogenous Notch1 in its nuclear form, which has oncogenic functions ${ }^{19,20}$. We immunoprecipitated lysates of MOLT- 4 cells with an antibody to endogenous ATM and observed a specific interaction with endogenous Notch1 (Fig. 3c). CUTLL1 cell lysates that were similarly immunoprecipitated with an N1IC-specific antibody confirmed the interaction between endogenous ATM and Notch1 (Fig. 3d). Thus, endogenous Notch1 and ATM also form a complex in Notch-driven cancer cells.

To independently validate this interaction, we used the in situ proximity ligation assay (PLA) to visualize the Notch1-ATM protein complex in individual nuclei of living cells. Cells transfected with $\mathrm{N} 1 \Delta \mathrm{E}$, but not those transfected with empty vector, showed bright fluorescent-dot signals indicative of the Notch1-ATM complex
(Supplementary Fig. 3f). We also observed formation of dotted foci indicative of Notch1-ATM complexes in CUTLL1 cells expressing endogenous constitutively active nuclear Notch1, and GSI treatment reduced such signals (Fig. 3e,f).

Notch1 inactivates ATM by binding to the FATC domain of ATM To determine which domain of ATM protein binds Notch1, we incubated 12 consecutive glutathione S-transferase (GST)-fusion fragments spanning the entire ATM protein ${ }^{21}$ with lysates of HEK $293 \mathrm{~T}$ cells expressing $\mathrm{N} 1 \Delta \mathrm{E}$. We observed that $\mathrm{N} 1 \Delta \mathrm{E}$ preferentially binds to the 11th fragment of ATM (residues 2842-3056), which contains the FRAP-ATM-TRRAP-C-terminal (FATC) domain (Fig. 4a). This domain has previously been reported to have a regulatory role in the kinase activity of ATM $^{22}$.

We next tested whether the protein interaction between ATM and Notch1 was direct by incubating GST-ATM fragments with recombinant purified human N1IC. We observed that purified N1IC binds the 11th fragment of ATM but not other GST-fusion constructs (Fig. 4b). Thus, the ATM-Notch1 interaction is direct and specific for a domain of ATM involved in regulating its kinase activity.

We also determined which domain in Notch1 is responsible for its interaction with ATM. The ankyrin domain is a large protein-protein-interaction surface at the $\mathrm{N}$ terminus of activated Notch1. We deleted ankyrin repeats in the N1 $\Delta$ E-Flag expression construct, generating N1 $\Delta \mathrm{E} \Delta \mathrm{ANK}$-Flag. Upon its expression in HEK293T cells, we observed that whereas N1 $\Delta$ E bound ATM, binding of N $1 \Delta \mathrm{E} \Delta \mathrm{ANK}$ was impaired (Supplementary Fig. 4a). We also observed a reduction in pATM foci in $\mathrm{N} 1 \Delta \mathrm{E}$-expressing cells, which was lost in N1 $\Delta \mathrm{E} \Delta \mathrm{ANK}$-expressing cells (Supplementary Fig. $4 \mathbf{b}$ ). Because Notch1-ATM binding is not completely impaired in the absence of the ankyrin domain, and a fraction of cells still can form foci in the presence of $\mathrm{N} 1 \Delta \mathrm{E} \Delta \mathrm{ANK}$, an additional domain in the Notch 1 protein may contribute to the interaction between Notch1 and ATM.

Finally, we tested whether Notch1 binding to ATM affects its kinase activity, by using a well-established in vitro system, cell-free extract from X. laevis eggs ${ }^{23}$. When linear double-stranded DNA is added 
to these extracts, activation of the DDR can be monitored by $\mathrm{pS} / \mathrm{TQ}$ and pATM signals, which are detectable by immunoblotting (Fig. 4c). Strikingly, addition of purified recombinant human Notch1 to the same DNA-containing extracts strongly prevented activation of ATM (pATM) and the phosphorylation of its substrates (pS/TQ), but it did not affect the unrelated kinase pERK (Fig. 4c). These results also confirm that ATM inactivation does not require Notch 1 transcriptional activity because there was no de novo transcription in $X$. laevis extracts.

Finally, we tested the effect of recombinant purified human Notch 1 on the kinase activity of affinity-purified human ATM $^{24}$. We immunoprecipitated ATM from lysates of HeLa cells to high purity (Supplementary Fig. 4c), and ATM in vitro kinase assays showed that GST-p53 is phosphorylated on Ser15 upon addition of doublestranded DNA. Such phosphorylation is progressively inhibited upon incubation with increasing amounts of purified recombinant human Notch1 (Fig. 4d and Supplementary Fig. 4d). We conclude that Notch 1 directly binds to the regulatory FATC domain of ATM and specifically inhibits its kinase activity.

Notch1 inhibition increases ATM-dependent apoptosis in T-ALL Notch 1 has a causative oncogenic role in T-ALL ${ }^{9}$. Over $50 \%$ of people with T-ALL carry activating mutations in Notch1 (ref. 25). We chose the TALL-1 cell line to address the physiological role of Notch1 interaction with ATM. We hypothesized that in these cells Notch1 may prevent IR-induced apoptosis by preventing ATM activation. To test this, we pretreated TALL-1 cells with GSI to reduce Notch1 activation, as confirmed by decreased levels of Hes1 (Supplementary Fig. 5a), and after exposing cells to IR, we performed immunoblotting for cleaved caspase-3, a marker of apoptosis. We observed that cells pretreated with GSI showed higher levels of apoptosis, at earlier time points (Fig. 5a). The observed increased apoptosis was ATM dependent because a specific small-molecule ATM kinase-activity inhibitor (Supplementary Fig. 5b) prevented it (Fig. 5a). Furthermore, the increase in DDR signaling after exposure to IR precedes apoptosis, as shown on the immunoblot for pATM and pCHK2T68 (Fig. 5b,c). The efficacy of GSI is confirmed by the decreased levels of Hes 1 (Supplementary Fig. 5c). By following the cells treated as above over time, we observed that the combination of GSI and IR leads to compromised survival of TALL-1 cells and that this can be rescued by ATM inhibition (Fig. 5d,e). We conclude that Notch1 inhibition in leukemic cells enhances DNA damage-induced cell death in an ATM-dependent manner.

\section{Notch1 represses ATM activation in breast cancer}

Aberrant Notch1 signaling has been widely reported in breast cancer ${ }^{26}$. To extend our analyses beyond T-ALL and to seek further validation of an inhibitory role of Notch1 on ATM activity, we analyzed activated Notch1 and PATM in a set of human primary breast cancer tissues. We observed that both activated Notch 1 and pATM were detectable in the cell nuclei of these tumors. We detected strong staining for activated Notch1 in 35\% of breast cancer cases studied, which was preferentially associated with high-grade tumors, results in agreement with previous studies ${ }^{13,27}$. In contrast, high expression of pATM (24\% of tumor samples) was mainly associated with low-grade breast tumors (Fig. 6a and Supplementary Fig. 6a). Remarkably, activated Notch1 and pATM levels showed a statistically significant inverse correlation $(P<0.00001)$ in these tumor samples (Fig. 6b). Because tumors may have different levels of $\mathrm{ATM}^{28}$, we excluded from this analysis the relatively small fraction of tumors (7\%) that showed undetectable levels of ATM (Supplementary Fig. 6b). An inverse correlation between activated Notch1 and PATM persisted in this set of breast cancers as well ( $P<0.009343$; Supplementary Fig. 6c). Next, we scored ATM expression levels as intermediate level (score 2) and highest level (score 3). The inverse correlation between Notch1 and pATM was maintained $(P<0.001021$ (score 2$)$ and $P<0.009343$ (score 3); Fig. 6c). When we analyzed individual breast cancer molecular subtypes, we found significantly higher levels of activated Notch1, particularly in high-grade breast tumors such as those of TN and HER2 ${ }^{+}$ subtype $(P<0.004064$; Supplementary Fig. 6d). These same subtypes showed low levels of pATM $(P<0.0536)$.

To support the results obtained by immunohistochemistry (IHC) analyses of breast cancers, we investigated Notch1 and ATM activation in a metadata set of 25 independent breast cancer gene-expression studies, collectively consisting of 3,661 human primary breast cancer samples (Online Methods and Supplementary Table 1). We registered the activity of Notch 1 by using the Notch 1 direct target gene signature (NDT), composed of Notch1 targets and exhibiting validated responsiveness to Notch1, direct promoter binding and expression in breast cancer $^{29}$. Similarly, we used an ATM-activity signature, composed of genes activated by DNA damage in an ATM-dependent manner in human embryonic kidney cells $\mathrm{s}^{30}$ and in mouse lymphoid tissue ${ }^{31}$ to recapitulate activated pATM (Supplementary Table 2 and Online Methods). We used gene-set enrichment analysis (GSEA) ${ }^{32}$ to search for a statistical association between the ATM-activity signature and the NDT signature. When comparing tumor samples expressing high or low levels of activated Notch1, we found that the ATM-activity signature was significantly overrepresented among downregulated genes; i.e., the ATM-activity signature was upregulated in patients with low levels of the NDT signature (false discovery rate of 0.015 ; Fig. 6d).

Patients with inversely correlated levels of NDT and ATM-activity signatures (i.e., patients classified as having low NDT and high ATM-activity signatures; or high NDT and low ATM-activity signatures) were significantly over-represented ( $P=0.002$ by one-sided Fisher's exact test). Finally, samples classified with high NDT and low ATM-activity signatures showed significantly poorer patient-survival probability by univariate Kaplan-Meier analysis $\left(P=6.77 \times 10^{-6}\right.$; Supplementary Fig. 6e).

Therefore, similarly to results in the C. elegans germline and in cultured cells, Notch 1 activity and ATM activation are also inversely correlated in human breast cancers, as independently concluded by analyses with IHC and expression microarray data sets.

\section{DISCUSSION}

Our results unveil for the first time, to our knowledge, an interaction between the Notch signaling pathway and the DDR apparatus via the apical kinase ATM. The gonad of C. elegans is a powerful biological system to study the role of Notch signaling at the endogenous level because the germ stem cells in the mitotic compartment of this organ rely on the activity of Notch-GLP-1 receptor for their proliferation ${ }^{33}$. In our analyses, we observed very low levels of ATM activation in the mitotic germ cells compared to the meiotic cells within the same gonad. Through genetic approaches, we found that C. elegans mutant strains carrying activating or inactivating DNA mutations in the $g l p-1$ gene display impaired or enhanced ATM activation, respectively, within the germline. This is in agreement with the well-documented antiapoptotic role of Notch signaling in mammalian cells in vitro ${ }^{16}$ and in Notch-driven cancers ${ }^{34-37}$.

Intriguingly, when exposed to DNA damage, cells in the worm mitotic compartment arrest in proliferation but fail to undergo apoptosis despite having detectable levels of p53 (refs. 38,39). At present, it is unclear why apoptosis is not triggered, but prevention of the apoptotic process may be a consequence of attenuated ATM activation in mitotic 
cells. Alternatively, DDR activation in stem cells may cause their differentiation, as we and others have previously shown ${ }^{40,41}$.

Importantly, the effect of Notch on ATM is evolutionarily conserved. Human cells expressing Notch1 show inactivation of ATM and other DDR components ${ }^{42}$. Unexpectedly, this effect of Notch 1 on ATM occurs in a transcription-independent manner. Indeed, we found that Notch1 binds to ATM through a direct interaction with the FATC domain of ATM. This domain has a crucial role in the activation of the kinase, as previously demonstrated in vitro ${ }^{22}$. Importantly, amino acid substitutions in the FATC domain are associated with increased predisposition to breast cancer ${ }^{43}$.

Strong support of the inhibitory function of Notch1 on ATM activation comes from results of the kinase assays performed in X. laevis egg extracts in which recombinant purified Notch1 inhibits the kinase activity of ATM. The support for a direct role of ATM inhibition by Notch 1 is further provided by the results showing the inhibitory activity of purified human Notch1 with immunopurified human ATM. In addition to strengthening the evolutionary conservation of the observations made in C. elegans, X. laevis and humans, the lack of transcription in these in vitro systems further supports a transcriptionindependent activity of Notch1 in ATM inhibition.

We also show that inhibition of Notch1 by GSI in the presence of DNA damage leads to increased radiosensitivity in an ATM-dependent manner in TALL-1 cells. Research in Notch inhibition is currently being pursued for its perceived value in treating cancer ${ }^{44}$. Here we provide evidence that cancer cells treated with DNA-damaging agents may undergo more robust cell death if treated with a Notch inhibitor, and we show that the observed increase in apoptosis is ATM dependent. This indicates a new use for Notch inhibitors as modulators of the response to DNA damage and also suggests that DDR proficiency (such as the ability to activate ATM) is an important factor to consider for an efficient therapeutic response to Notch inhibition. Finally, the relevance of our results for cancer studies is further indicated by the observed inverse correlation between Notch1 and ATM activation in human breast cancers, as concluded by two independent approaches. The progressive increase in Notch1 expression from low- to high-grade tumors is consistent with the idea that Notch1 signaling is activated in breast cancer and that Notch 1 may have an important role in tumor progression ${ }^{45}$. Conversely, low-grade tumors showing ATM activation to a greater extent than high-grade ones supports a model in which DDR acts as a barrier to tumor progression ${ }^{46,47}$.

A growing body of evidence has suggested that Notch1 not only plays a part in tumor progression but also is important for chemoresistance, metastasis dissemination and cancer stem-cell biology ${ }^{29}$. In this regard, the observation that stem cells can undergo cell differentiation upon DNA damage in an ATM-dependent manner, and that this may also apply to cancers $^{41}$, provides a tantalizing potential link between cancer stem-cell maintenance and the need for Notch activity. Our results may contribute to a better characterization of a subset of human breast tumors and guide new therapeutic treatments.

\section{METHODS}

Methods and any associated references are available in the online version of the paper.

Note: Any Supplementary Information and Source Data files are available in the online version of the paper.

\section{ACKNOWLEDGMENTS}

We thank T. Schedl (Washington University School of Medicine), M. Donzelli (Istituto Europeo di Oncologia), P.P. Di Fiore (Istituto Europeo di Oncologia), N. Offenhaeuser (Istituto Fondazione Italiana per la Ricerca sul Cancro di
Oncologia Molecolare), S. Pece (Istituto Europeo di Oncologia), J.C. Zuniga-Pflucker (Sunnybrook and Women's College Health Sciences Center), P.G. Pelicci (Istituto Europeo di Oncologia), A. Sarin (National Centre for Biological Sciences), A. Behrens (London Research Institute), T. Halazonetis (University of Geneva), A. Ferrando (Columbia University) and W.S. Pear (Abramson Family Cancer Research Institute) for reagents; T. Vaccari, F. Kobia, A. Petrovic and

A. Musacchio for advice; G. Bottai and G. Bianchini for technical assistance with IHC experiments; and S. Pasqualato and S. Monzani for help with purification of human recombinant Notch1. L.S. is supported by a grant from the Associazione Italiana per la Ricerca sul Cancro (AIRC; grant 6251). S.B. and M.F. are supported by funds from the AIRC Special Program Molecular Clinical Oncology ' 5 per mille’ and from Fondo per gli Investimenti della Ricerca di Base (FIRB; Accordi di Programma 2011 RBAP11T3WB). G.D.S. is supported by grants from the AIRC Special Program Molecular Clinical Oncology ' 5 per mille' and from the Italian University and Research Ministerium (Cofin FIRB). F.d'A.d.F. is supported by the Fondazione Italiana per la Ricerca sul Cancro, AIRC (application 12971), Human Frontier Science Program (contract RGP 0014/2012), Cariplo Foundation (grant 2010.0818), Marie Curie Initial Training Networks (FP7 PEOPLE 2012 ITN (CodAge)), Fondazione Telethon (GGP12059), Association for International Cancer Research, Progetti di Ricerca di Interesse Nazionale (PRIN) 2010-2011, the Italian Ministry of Education Universities and Research EPIGEN Project, and an European Research Council advanced grant (322726).

\section{AUTHOR CONTRIBUTIONS}

F.d'A.d.F. and J.V. designed the study. J.V., M.A., L.S., M.F. and A.R. performed the experiments. J.V., M.A., L.S., M.F., S.B., A.R., G.D.S., V.C. and F.d'A.d.F. analyzed the data. L.M. performed baculovirus infections of insect cells, and C.L. helped with coculture of OP9-DL1 cells. F.d'A.d.F. and J.V. wrote the paper. All authors discussed the results and commented on the manuscript.

\section{COMPETING FINANCIAL INTERESTS}

The authors declare no competing financial interests.

Reprints and permissions information is available online at http://www.nature.com/ reprints/index.html.

1. d'Adda di Fagagna, F. Living on a break: cellular senescence as a DNA-damage response. Nat. Rev. Cancer 8, 512-522 (2008).

2. Jackson, S.P. \& Bartek, J. The DNA-damage response in human biology and disease. Nature 461, 1071-1078 (2009).

3. Bailly, A. \& Gartner, A. Germ cell apoptosis and DNA damage responses. Adv. Exp. Med. Biol. 757, 249-276 (2013).

4. Stergiou, L. \& Hengartner, M.O. Death and more: DNA damage response pathways in the nematode C. elegans. Cell Death Differ. 11, 21-28 (2004).

5. Ntziachristos, P., Lim, J.S., Sage, J. \& Aifantis, I. From fly wings to targeted cancer therapies: a centennial for Notch signaling. Cancer Cell 25, 318-334 (2014).

6. Andersson, E.R., Sandberg, R. \& LendahI, U. Notch signaling: simplicity in design, versatility in function. Development 138, 3593-3612 (2011).

7. Andersen, P., Uosaki, H., Shenje, L.T. \& Kwon, C. Non-canonical Notch signaling: emerging role and mechanism. Trends Cell Biol. 22, 257-265 (2012).

8. Koch, U. \& Radtke, F. Notch and cancer: a double-edged sword. Cell. Mol. Life Sci. 64, 2746-2762 (2007).

9. Ellisen, L.W. et al. TAN-1, the human homolog of the Drosophila notch gene, is broken by chromosomal translocations in T lymphoblastic neoplasms. Cell 66, 649-661 (1991).

10. Vermezovic, J., Stergiou, L., Hengartner, M.O. \& d'Adda di Fagagna, F. Differential regulation of DNA damage response activation between somatic and germline cells in Caenorhabditis elegans. Cell Death Differ. 19, 1847-1855 (2012).

11. Pepper, A.S., Lo, T.W., Killian, D.J., Hall, D.H. \& Hubbard, E.J. The establishment of Caenorhabditis elegans germline pattern is controlled by overlapping proximal and distal somatic gonad signals. Dev. Biol. 259, 336-350 (2003).

12. Kodoyianni, V., Maine, E.M. \& Kimble, J. Molecular basis of loss-of-function mutations in the g/p-1 gene of Caenorhabditis elegans. Mol. Biol. Cell 3, 1199-1213 (1992)

13. Rustighi, A. et al. The prolyl-isomerase Pin 1 is a Notch 1 target that enhances Notch1 activation in cancer. Nat. Cell Biol. 11, 133-142 (2009).

14. Rand, M.D. et al. Calcium depletion dissociates and activates heterodimeric notch receptors. Mol. Cell. Biol. 20, 1825-1835 (2000).

15. Schmitt, T.M. \& Zuniga-Pflucker, J.C. Induction of $T$ cell development from hematopoietic progenitor cells by delta-like-1 in vitro. Immunity 17, 749-756 (2002).

16. Perumalsamy, L.R., Nagala, M., Banerjee, P. \& Sarin, A. A hierarchical cascade activated by non-canonical Notch signaling and the mTOR-Rictor complex regulates neglect-induced death in mammalian cells. Cell Death Differ. 16, 879-889 (2009).

17. Borggrefe, T. \& Oswald, F. The Notch signaling pathway: transcriptional regulation at Notch target genes. Cell. Mol. Life Sci. 66, 1631-1646 (2009).

18. Westhoff, B. et al. Alterations of the Notch pathway in lung cancer. Proc. Natl. Acad. Sci. USA 106, 22293-22298 (2009). 
19. Palomero, T. et al. CUTLL1, a novel human T-cell lymphoma cell line with $t(7 ; 9)$ rearrangement, aberrant $\mathrm{NOTCH} 1$ activation and high sensitivity to gamma-secretase inhibitors. Leukemia 20, 1279-1287 (2006).

20. Weng, A.P. et al. Activating mutations of $\mathrm{NOTCH} 1$ in human $\mathrm{T}$ cell acute lymphoblastic leukemia. Science 306, 269-271 (2004).

21. Khanna, K.K. et al. ATM associates with and phosphorylates p53: mapping the region of interaction. Nat. Genet. 20, 398-400 (1998).

22. Jiang, X., Sun, Y., Chen, S., Roy, K. \& Price, B.D. The FATC domains of PIKK proteins are functionally equivalent and participate in the Tip60-dependent activation of DNA-PKCS and ATM. J. Biol. Chem. 281, 15741-15746 (2006).

23. Garner, E. \& Costanzo, V. Studying the DNA damage response using in vitro model systems. DNA Repair (Amst.) 8, 1025-1037 (2009).

24. Smith, G.C. et al. Purification and DNA binding properties of the ataxia-telangiectasia gene product ATM. Proc. Natl. Acad. Sci. USA 96, 11134-11139 (1999).

25. Grabher, C., von Boehmer, H. \& Look, A.T. Notch 1 activation in the molecular pathogenesis of T-cell acute lymphoblastic leukaemia. Nat. Rev. Cancer 6, 347-359 (2006).

26. Stylianou, S., Clarke, R.B. \& Brennan, K. Aberrant activation of notch signaling in human breast cancer. Cancer Res. 66, 1517-1525 (2006).

27. Farnie, G. et al. Novel cell culture technique for primary ductal carcinoma in situ: role of Notch and epidermal growth factor receptor signaling pathways. J. Natl. Cancer Inst. 99, 616-627 (2007).

28. Bisso, A. et al. Oncogenic miR-181a/b affect the DNA damage response in aggressive breast cancer. Cell Cycle 12, 1679-1687 (2013).

29. Rustighi, A. et al. Prolyl-isomerase Pin 1 controls normal and cancer stem cells of the breast. EMBO Mol. Med. 6, 99-119 (2014).

30. Elkon, R. et al. Dissection of a DNA-damage-induced transcriptional network using a combination of microarrays, RNA interference and computational promoter analysis. Genome Biol. 6, R43 (2005).

31. Rashi-Elkeles, S. et al. Parallel induction of ATM-dependent pro- and antiapoptotic signals in response to ionizing radiation in murine lymphoid tissue. Oncogene 25, 1584-1592 (2006).

32. Subramanian, A. et al. Gene set enrichment analysis: a knowledge-based approach for interpreting genome-wide expression profiles. Proc. Natl. Acad. Sci. USA 102, 15545-15550 (2005)
33. Kimble, J. \& Crittenden, S.L. Controls of germline stem cells, entry into meiosis, and the sperm/oocyte decision in Caenorhabditis elegans. Annu. Rev. Cell Dev. Biol. 23, 405-433 (2007).

34. Fassl, A. et al. Notch1 signaling promotes survival of glioblastoma cells via EGFRmediated induction of anti-apoptotic Mcl-1. Oncogene 31, 4698-4708 (2012).

35. Wang, J. et al. Notch promotes radioresistance of glioma stem cells. Stem Cells 28, 17-28 (2010).

36. Sade, H., Krishna, S. \& Sarin, A. The anti-apoptotic effect of Notch-1 requires p56lck-dependent, Akt/PKB-mediated signaling in T cells. J. Biol. Chem. 279, 2937-2944 (2004).

37. Beverly, L.J., Felsher, D.W. \& Capobianco, A.J. Suppression of p53 by Notch in lymphomagenesis: implications for initiation and regression. Cancer Res. 65, 7159-7168 (2005).

38. Gartner, A., Milstein, S., Ahmed, S., Hodgkin, J. \& Hengartner, M.O. A conserved checkpoint pathway mediates DNA damage-induced apoptosis and cell cycle arrest in C. elegans. Mol. Cell 5, 435-443 (2000).

39. Schumacher, B., Hofmann, K., Boulton, S. \& Gartner, A. The C. elegans homolog of the p53 tumor suppressor is required for DNA damage-induced apoptosis. Curr. Biol. 11, 1722-1727 (2001).

40. Wang, J. et al. A differentiation checkpoint limits hematopoietic stem cell selfrenewal in response to DNA damage. Cell 148, 1001-1014 (2012).

41. Schneider, L. et al. DNA damage in mammalian neural stem cells leads to astrocytic differentiation mediated by BMP2 signaling through JAK-STAT. Stem Cell Reports 1, 123-138 (2013)

42. Kim, S.B. et al. Activated Notch1 interacts with p53 to inhibit its phosphorylation and transactivation. Cell Death Differ. 14, 982-991 (2007).

43. Tavtigian, S.V. et al. Rare, evolutionarily unlikely missense substitutions in ATM confer increased risk of breast cancer. Am. J. Hum. Genet. 85, 427-446 (2009).

44. Groth, C. \& Fortini, M.E. Therapeutic approaches to modulating Notch signaling: current challenges and future prospects. Semin. Cell Dev. Biol. 23, 465-472 (2012).

45. Politi, K., Feirt, N. \& Kitajewski, J. Notch in mammary gland development and breast cancer. Semin. Cancer Biol. 14, 341-347 (2004).

46. Campisi, J. \& d'Adda di Fagagna, F. Cellular senescence: when bad things happen to good cells. Nat. Rev. Mol. Cell Biol. 8, 729-740 (2007).

47. Halazonetis, T.D., Gorgoulis, V.G. \& Bartek, J. An oncogene-induced DNA damage model for cancer development. Science 319, 1352-1355 (2008). 


\section{ONLINE METHODS}

C. elegans culture conditions and strains. C. elegans strains were cultured and maintained according to standard procedures ${ }^{48}$. N2 and $g l p-1$ (q224)III strains were provided by the Caenorhabditis Genetics Centre (University of Minnesota). Strain $g l p-1$ (ar202) was a kind gift from T. Schedl (Washington University School of Medicine).

C. elegans immunostaining and in situ RNA hybridization. Immunostaining on isolated gonads was performed as described previously ${ }^{10}$. Anti-pS/TQ (Cell Signaling) was used at 1:100 dilution. For RNA in situ hybridization, dissected gonads were fixed in $0.25 \%$ glutaraldehyde, $3 \%$ formaldehyde and $100 \mathrm{mM}$ $\mathrm{K}_{2} \mathrm{HPO}_{4}, \mathrm{pH} 7.2$, and processed as previously described ${ }^{49,50}$. Sense and antisense DNA probes for atm-1 and gld-1 were synthesized with digoxigenin-11-dUTP by repeated primer extension. At least 10 gonads per strain were analyzed.

C. elegans imaging and treatments. Confocal sections were obtained with a Leica TCS SP2 AOBS confocal laser microscope by sequential scanning. Regions of interest were converted into maximal-intensity projections with ImageJ software. Close-up images of pachytene regions in the C. elegans germline were acquired with a DeltaVision Elite deconvolution microscope (Applied Precision). Stacks were deconvoluted with Delta Vision SoftWoRx. X-rays were generated by a radiationgenerating machine (Faxitron X-Ray Corporation). Worms were treated with $180 \mathrm{~Gy}$ of IR and analyzed $1 \mathrm{~h}$ after treatment.

Cell culture and treatments. HeLa (ATCC) and HEK293T (ICLC) cells were grown under standard tissue-culture conditions. TALL-1 (DSMZ), CUTLL1 (provided by A. Ferrando (Columbia University)) and MOLT-4 (ATCC) cells were cultured in RPMI supplemented with 10\% FBS. MCF10a (ATCC) cells were grown in DMEM/F12 (1:1), $5 \%$ horse serum, $0.5 \mathrm{mg} / \mathrm{ml}$ hydrocortisone, $10 \mu \mathrm{g} / \mathrm{ml}$ insulin, $100 \mathrm{ng} / \mathrm{ml}$ cholera toxin and $20 \mathrm{ng} / \mathrm{ml} \mathrm{EGF}$. OP9-DL1 (kindly provided by J.C. Zuniga-Pflucker (Sunnybrook and Women's College Health Sciences Center)) cells were grown as described previously ${ }^{51}$. Endogenous Notch1 was induced in MCF10a cells with a 10-min treatment of $10 \mathrm{mM} \mathrm{EGTA.} \mathrm{Cells} \mathrm{were} \mathrm{then} \mathrm{rinsed,}$ and fresh complete medium was added. Cells were then exposed to $0.5 \mathrm{~Gy}$ of IR and collected after $20 \mathrm{~min}$ for analysis. For the G1/S-checkpoint assay, Notch was induced in MCF10a cells by EGTA as above. Cells were exposed to $10 \mathrm{~Gy}$ IR, and this was followed by a 30-min pulse of BrdU, before fixation. Infections of MCF10a or HeLa cells were performed with standard protocols for retroviral transduction. Infected cells were sorted with FACS for a GFP marker $1 \mathrm{~d}$ after infection, replated after sorting and exposed to 5 Gy of IR. Protein lysates were prepared $1 \mathrm{~h}$ after IR. DAPT ( $N$-[N-(3,5-difluorophenacetyl)-L-alanyl $]-S$ phenylglycine $t$-butyl ester, Sigma) was used at $10 \mu \mathrm{M}$ concentration in an overnight treatment of HeLa cells. Gamma-secretase inhibitor I (GSI-I, $t$-3,5-DMCIL-CHO or N-trans-3,5-dimethoxycinnamoyl)-Ile-leucinal, Merck Chemicals) was used at $10 \mu \mathrm{M}$ concentration for $3 \mathrm{~h}$ in a treatment of TALL- 1 cells. KU55933 (ATM inhibitor, 2-(4-morpholinyl)-6-(1 thianthrenyl)-4H-pyran-4-one, Tocris Bioscience) was used at $10 \mu \mathrm{M}$ concentration for $3 \mathrm{~h}$ in a treatment of TALL- 1 cells. $\mathrm{X}$-rays were generated by a radiation-generating machine (Faxitron X-Ray Corporation), and doses are indicated in the figure legends. HeLa cells were treated with neocarzinostatin (NCS) at $50 \mathrm{ng} / \mathrm{ml}$ for $10 \mathrm{~min}$ and then washed. Cells were collected $50 \mathrm{~min}$ after.

Cell cocultures. Cells were cocultured in 24-well plates. MCF10a cells were plated at $20 \times 10^{3}$ cells/well on glass coverslips. $48 \mathrm{~h}$ later, $50 \times 10^{3}$ cells/well of OP9/OP9-DL1 cells were added onto a monolayer of MCF10a cells. Cells were cocultured for $2.5 \mathrm{~h}$ before exposure to IR $(0.5 \mathrm{~Gy}) .0 .5 \mathrm{~h}$ later, cells were fixed in paraformaldehyde (PFA) for $10 \mathrm{~min}$ for immunostaining.

Immunostaining of mammalian cells. Cells were fixed in $50 \%$ methanol and $50 \%$ acetone for $2 \mathrm{~min}$ at room temperature. Coverslips were blocked in PBG $(0.2 \%$ cold-water-fish gelatin and $0.5 \%$ BSA in PBS) and incubated with primary antibody. Cells were washed and incubated with secondary antibodies (Jackson Laboratories), and nuclei were counterstained with DAPI. Images were acquired with a wide-field Olympus Biosystems Microscope and MetaVue software. Comparative immunofluorescence microscopy analyses were performed in parallel with identical acquisition and analysis parameters. A minimum of 100 and a maximum of 3,000 cells were scored for quantification, depending on the experiment. Quantification of nuclear foci and their average intensities per nucleus in MCF10a cells was performed with automated image-analysis software (Cellprofiler 2.0 (ref. 52)) on images acquired with a 20× dry objective (Olympus UPlanFL N, $0.5 \mathrm{NA}$ ).

In situ proximity ligation assay (PLA, Duolink). Cells were labeled according to the manufacturer's instructions (Sigma). Briefly, HeLa cells expressing N1 $\Delta \mathrm{E}$ were fixed in methanol/acetone for $2 \mathrm{~min}$ at room temperature. After incubation with primary antibodies, appropriate PLA probes (secondary antibodies conjugated with oligonucleotides) were added to the samples. Ligation of the oligonucleotide probes that were in proximity (less than $40 \mathrm{~nm}$ ) was then performed, after which fluorescently labeled oligonucleotides were added together with a DNA polymerase to generate a signal detectable by a fluorescence microscope.

Prior to Duolink staining, CUTLL1 cells were treated with $1 \mu \mathrm{M}$ compound E (Calbiochem) for $48 \mathrm{~h}$. Cells were then fixed in 2\% PFA for $20 \mathrm{~min}$ at room temperature. After being washed with PBS, cells were placed on polylysinecoated slides and subjected to a cytospin concentration (800 r.p.m., 5 min). Quantification of nuclear Notch1-ATM dots was performed with automated image-analysis software (Cell profiler 2.1.0)

Transfections. Transient transfections in HeLa cells were performed with Lipofectamine (Invitrogen). Cells were collected for analysis $24 \mathrm{~h}$ after transfection. HEK293T cells were transfected with a standard calcium phosphate method and collected $24 \mathrm{~h}$ after the transfection.

qPCR. Total RNA was isolated from cells by TRIzol (Invitrogen)-chloroform extraction. CDNA was generated with the VILO kit according to the manufacturer's protocol (Invitrogen). The cDNA was used as a template in quantitative PCR reactions with specific primers on a Roche LightCycler 480 Sequence Detection System. The reactions were prepared with SYBR Green reaction mix from Roche. Ribosomal protein P0 (RPP0) was used as a control gene for normalization. Primer sequences for qRT-PCR were: RPPO-fw, 5'-TTCATTGTGGGAGCAGAC-3'; RPPO-rev, 5'-CAGCAGTTTCTCCAGAGC-3'; HES1-fw, 5'-GAAGCACCTCCGGAACCT-3'; and HES1-rv, 5'-GTCACCTCGTTCATGCACTC-3'.

Cloning and production of recombinant human Notch1. Human N1IC-Flag was generated by PCR with pCDNA3-N1 $\Delta$ E-Flag as a template. The N1IC-Flag PCR fragment was subcloned into the BamHI/SalI site of pFastBacl (Invitrogen). The MultiBac expression system (Invitrogen) was used as described previously ${ }^{53}$, for protein expression in $\mathrm{Hi} 5$ insect cells. Cells expressing N1IC-Flag were lysed (20 mM Tris- $\mathrm{HCl}, \mathrm{pH} 8,300 \mathrm{mM} \mathrm{NaCl}, 4 \mathrm{mM} \mathrm{MgCl}_{2}, 0.4 \mathrm{mM}$ EDTA and 20\% glycerol), and cleared cell lysates were incubated with anti-Flag M2 beads (Sigma). Recombinant N1IC-Flag fusion protein was eluted from the beads by competition with Flag peptide (Sigma).

Immunoprecipitations and immunoblotting. Cell extracts were prepared by lysis in TEB150 buffer (50mM HEPES, pH 7.4, $150 \mathrm{mM} \mathrm{NaCl}, 2 \mathrm{mM} \mathrm{MgCl}$, 5 mM EGTA, pH 8, 1 mM dithiothreitol (DTT), 0.5\% Triton X-100, 10\% glycerol, $1 \mu \mathrm{M}$ microcystin-LR (Enzo Lifesciences), protease-inhibitor cocktail set III (Calbiochem) and benzonase nuclease (Sigma)). Proteins were resolved by SDS-PAGE, transferred to nitrocellulose membrane and probed with the appropriate primary antibodies. For immunoprecipitations, cell lysates in TEB150 buffer were cleared with protein G-Sepharose (Zymed Laboratories). Binding reactions were performed overnight at $4{ }^{\circ} \mathrm{C}$ and were followed by addition of protein G-Sepharose beads for $2 \mathrm{~h}$. Nonrelated antibodies used as controls in IPs with endogenous proteins were against mouse IgG and myc. Beads were then washed and resuspended in a sample buffer before immunoblotting. For ethidium bromide $(\mathrm{EtBr})$ and DNase treatments, lysates of HEK293T cells were subjected to 1 -h treatment with $50 \mu \mathrm{g} / \mathrm{ml}$ EtBr or DNase (2 U) for $10 \mathrm{~min}$.

Pulldowns and in vitro binding assays. For pulldown reactions, purified GST-ATM fragments were immobilized on glutathione-Sepharose 4B beads (Amersham Biochemistry). Immobilized ATM fragments were incubated with lysates of N1 $\Delta$ E-expressing HEK293T cells in TEB150 buffer at $4{ }^{\circ} \mathrm{C}$ for $2 \mathrm{~h}$. Beads were then washed and resuspended in a sample buffer before being loaded on an SDS-PAGE gel and subjected to immunoblotting. For in vitro 
binding assays, $1 \mu \mathrm{g}$ of recombinant human N1IC-Flag and $1 \mu \mathrm{g}$ of each GST-ATM fragment were used for each reaction.

$X$. laevis egg extracts and kinase assay. Egg extracts were prepared as described previously ${ }^{54} .20 \mu \mathrm{l}$ of egg extracts were preincubated with recombinant purified human Notch1 $(320 \mathrm{nM})$ for $20 \mathrm{~min}$ at $23^{\circ} \mathrm{C}$; this was followed by 30 -min incubation with/without 20 ng of linear DNA (50-bp DNA Ladder, New England BioLabs). The extracts were then resuspended in Laemmli buffer and loaded on an SDS-PAGE gel.

ATM immunocomplex kinase assay. HeLa cells were harvested and lysed in TEB 150 (without phosphatase inhibitors) for $30 \mathrm{~min}$ on ice; this was followed by centrifugation at $16,000 \mathrm{~g}$ at $4{ }^{\circ} \mathrm{C}$ for $15 \mathrm{~min} .0 .5 \mathrm{mg}$ of the lysate was precleared with $30 \mu \mathrm{l}$ of Protein $\mathrm{G}$ beads $(1.5 \mathrm{~h})$; this was followed by incubation with antibodies to ATM $(0.5 \mu \mathrm{g}$ in $500 \mu \mathrm{l}$ volume, $1.5 \mathrm{~h})$ and addition of $30 \mu \mathrm{l}$ of protein $\mathrm{G}$ beads $(1.5 \mathrm{~h})$. Beads were centrifuged and washed sequentially with TEB 150 , TEB 150 with $0.5 \mathrm{M} \mathrm{LiCl}$, TEB 150 with $0.5 \mathrm{M} \mathrm{NaCl}$, twice with TEB 150 and once with kinase buffer (50 mM HEPES, pH 7.5, $50 \mathrm{mM} \mathrm{KCl} ; 5 \mathrm{mM} \mathrm{MgCl}_{2}$, $1 \mathrm{mM}$ DTT and $10 \%$ glycerol) for 5 min per wash. Next, immunoprecipitated ATM was incubated with recombinant Notch1 in kinase buffer; this was followed by addition of $30 \mathrm{nM}$ GST-p53, $10 \mathrm{ng}$ of DNA 1-kb ladder (NEB) and $1 \mathrm{mM}$ ATP. Kinase activity was allowed by incubation at $30^{\circ} \mathrm{C}$ for $90 \mathrm{~min}$. The reaction was stopped by addition of sample buffer and boiling.

GST-p53. Escherichia coli BL21 competent bacteria were transformed with pGex4T3-p53 plasmid. Expressed protein was purified with GSTrap (GE Healthcare).

Silver staining. Immunopurified ATM was run on an SDS-PAGE gel. The gel was fixed in fixing solution ( $40 \%$ methanol and $10 \%$ acetic acid) for $1 \mathrm{~h}$; this was followed by washes with $30 \%$ ethanol and water. The gel was further incubated for $1 \mathrm{~min}$ in a $0.03 \%$ solution of sodium thiosulfate and immersed in a $0.1 \%$ solution of silver nitrate for $20 \mathrm{~min}$ at $4{ }^{\circ} \mathrm{C}$. After washing of the gel, silver-stained proteins were developed with the use of cold developing solution ( $0.04 \%$ formaldehyde; $2 \%$ sodium carbonate). Reaction was stopped by addition of fixing solution.

Immunohistochemistry (IHC). Staining of activated Notch1 (ab8925, Abcam, 1:200), ATM pSer1981 (NB110-66655, Acris, 1:400) and total ATM (NB100-309, Novus Biologicals, 1:500) was performed according to standard procedures for paraffin-embedded tissue sections from 89 grade I-III invasive ductal breast carcinomas. The analyzed materials were de-identified stored human samples and tissues from deceased people. The study was approved by the Institutional Review Ethical Board at the Hospital of Prato, Istituto Toscano Tumori, Italy. No statistical method was used to predetermine sample size. The experiments were not randomized and were not performed with blinding to the conditions of the experiments. Slides were deparaffinized and rehydrated, and antigen was retrieved in a calibrated steam pressure cooker with citrate buffer ( $\mathrm{pH}$ 6.0). Endogenous peroxidase was blocked with peroxidase block from an EnVision Kit (DakoCytomation) for $15 \mathrm{~min}$. The slides were additionally immersed in 5\% nonfat dry milk for 20 min to minimize nonspecific binding due to hydrophobic interaction. The slides were then incubated with rabbit anti-Notch1 or anti-pATM antibody overnight at $4{ }^{\circ} \mathrm{C}$. Colorimetric detection was completed with diaminobenzidine and hydrogen peroxidase for $6 \mathrm{~min}$ and counterstained with hematoxylin (Sigma-Aldrich). The immunostaining was scored semiquantitatively. Only nuclear staining was considered in the evaluation of activated NOTCH-1 expression. The scores for IHC were: 0 , no staining; 1 , low nuclear staining of tumor cells ( $<5 \%$ of positive tumor cells); $2,5-10 \%$ of positive tumor cells; and 3, $>10 \%$ positive tumor cells. For pairwise comparisons, the scores were collapsed to low (score, 1 or 2 ) versus high (score, 3 ) expression, excluding noninterpretable samples.

Antibodies. Antibodies to pS/TQ (2851, Cell Signaling), CHK2 pT68 (2661, Cell Signaling), cleaved caspase-3 (Asp 175) (9661, Cell Signaling), DYKDDDDK tag (2368, Cell Signaling), cleaved Notch1 (Val1744) (clone D3B8, 4147, Cell Signaling), $\gamma$-H2AX (05-636, Millipore), CHK2 (05-649, Millipore), ATM pS1981 (200-301-400, Rockland), ATM pS1981 (ab81292, Abcam), ATM (clone MAT3-4G10/8, A1106, Sigma), vinculin (clone hVIN-1, V9131, Sigma), Flag (clone M2, F1804,
Sigma), $\alpha$-tubulin (clone B-5-1-2, T6074, Sigma), KAP1 pSer824 (A300-767, Bethyl), SMC1 pSer966 (A300-050, Bethyl), SMC1 (A300-055, Bethyl), Notch1(C20) (sc-6014, Santa Cruz), NBS1 (NB 100-143, Novus), nucleolin (NB 600-241, Novus), 53BP1 (NB100-304 Novus), GST (Biochemistry Facility, IFOM-IEO Campus), ATM (04-200, Millipore), KAP1 (ab10484, Abcam) and H2AX (ab11175, Abcam) were used. mAb 53BP1 was a kind gift from T. Halazonetis (University of Geneva). mAb 53BP1 was previously used for human cells ${ }^{55}$. Anti-Xenopus ATM was from V.C. and was characterized previously ${ }^{56}$. Validation information for all commercial antibodies is provided on their respective manufacturers' websites.

Plasmids. N1 $\triangle \mathrm{E} \triangle \mathrm{ANK}$ was generated by deletion of residues $1873-2082$ of the Notch1 ankyrin domain. pCDNA3-N1 $\Delta$ E-Flag was used as a template in a PCR reaction to amplify fragments surrounding the deletion. Two PCR fragments were subcloned into BamHI/EcoRV-EcoRV/XhoI sites of pCDNA3. pCDNA3N1 $\Delta$ E-Flag was a kind gift from P.P. Di Fiore (IEO). WT NOTCH-1 and NOTCH-1 $\triangle$ TAD were kindly provided by S. Pece (IEO). CD8-N1IC-GFP-, GFP-N1IC-NES- and GFP-N1IC-NLS-expressing vectors were a kind gift from A. Sarin (NCBS). Recombinant GST-ATM fusion proteins (1-12) were a kind gift from A. Behrens (London Research Institute) ${ }^{21}$. MSCV-IRES-GFP construct (MigR1) and N1IC/MigR1 were kindly provided by W. Pear (Abramson Family Cancer Research Institute). pGex4T3-p53 plasmid was a kind gift from P.G. Pelicci (IEO).

ATM-activity signature. The ATM-activity signature is composed of a selection of genes activated by DNA damage in an ATM-dependent manner in human embryonic kidney cells (HEK 293) ${ }^{30}$ and in mouse lymphoid tissue ${ }^{31}$. Elkon et al..$^{30}$ used microarrays to investigate the transcriptional response to treatment with the radiomimetic drug NCS in cells knocked down for ATM. Raw gene-expression data from ref. 30 were downloaded from Gene Expression Omnibus GSE1676. Expression values were generated with the robust multiarray average procedure $\left(\mathrm{RMA}^{57}\right)$ with custom-definition files for Affymetrix Human HG-Focus Target Arrays based on Entrez (Version 15; http://brainarray.mbni.med.umich.edu/ Brainarray/default.asp).

Differentially expressed genes were identified with the significance analysis of microarray (SAM) algorithm coded in the samr $R$ package ${ }^{58}$. In SAM, we estimated the percentage of false-positive predictions with 100 permutations and selected those transcripts whose false discovery rate was $\leq 0.05$. We selected genes upregulated in NCS-treated uninfected cells (as compared to untreated uninfected cells), upregulated in NCS-treated LacZ-knockdown cells (as compared to untreated LacZ-knockdown cells) and not upregulated in NCS-treated ATMknockdown cells (as compared to untreated ATM-knockdown cells). RashiElkeles et al. ${ }^{31}$ characterized the transcriptional response to ionizing radiation (IR) in mouse lymphoid tissue and identified an ATM-dependent response with wild-type and Atm-deficient mice. We obtained the gene list representing such response by merging clusters (cluster 1 and cluster 2 of the data from ref. 31), which contain ATM-dependent genes induced $30 \mathrm{~min}$ after IR treatment and late ATM-dependent genes induced by IR treatment, respectively. The intersection between the human and the mouse ATM-dependent gene lists resulted in a 14-gene signature composed of evolutionarily conserved genes representing ATM activity in DDR (Supplementary Table 2).

Breast cancer data collection and processing. We collected 27 data sets comprising microarray data of breast cancer samples and annotations on patients' clinical outcome. All data were measured on Affymetrix arrays and were downloaded from Gene Expression Omnibus (GEO) and ArrayExpress (http://www.ebi. ac.uk/arrayexpress/). Prior to analysis, we reorganized the data sets, eliminating duplicate samples and samples without outcome information, and we renamed any original study after the medical center where patients were recruited. This reorganization resulted in a metadata set comprising 3,661 unique samples from 25 independent cohorts (Supplementary Table 1). According to ref. 59, we standardized clinical information among the various data sets, redefining the outcome descriptions on the basis of the clinical annotations of each individual study. Specifically, we defined 'survival' as comprising overall survival, disease-free survival and disease-specific survival.

Raw expression data (i.e., CEL files) obtained from different platforms were integrated with an approach inspired by geometry and probe content of HG-U133 
Affymetrix arrays ${ }^{60}$. Briefly, probes with the same oligonucleotide sequence, but located at different coordinates on different types of arrays, may be arranged in a virtual platform grid. As for any other microarray geometry, this virtual grid may be used as a reference to create a virtual chip definition file (virtual CDF), containing the probes shared among the various HG-U133 platforms and their coordinates on the virtual platform, and virtual CEL files containing the fluorescence intensities of the original CEL files properly remapped on the virtual grid. Once we defined the virtual platform through the creation of the virtual CDF and transformed the CEL files into virtual CELs, the raw data, originally obtained from different HG-U133 arrays, were homogeneous in terms of platform and could be preprocessed and normalized with standard approaches, such as RMA. Specifically, expression values were generated from intensity signals with the virtual CDF obtained from merging HG-U133A, HG-U133AAofAV2, and HG-U133 Plus2 original CDFs and the transformed virtual-CEL files. Intensity values for a total of 21,981 meta-probe sets were background adjusted and normalized with quantile normalization, and gene expression levels were calculated with median polish summarization (RMA). The metadata set was corrected for batch effects with $\mathrm{ComBat}^{61}$. The entire procedure has been implemented as an $R$ script.

To identify two groups of tumor samples with either high or low levels of the Notch-dependent direct target (NDT) gene signature ${ }^{62}$, we used the classifier described in ref. 63. Briefly, we defined a classification rule based on summarizing the standardized expression levels of each gene in the NDT signature into a combined score with zero mean. Tumors were then classified as NDT-signature low if the combined score was negative and as NDT-signature high if the combined score was positive. The same classifier was used to identify two groups of tumors with either high or low ATM activity, summarizing the standardized expression levels of genes in the ATM-activity signature into a combined score with zero mean. Tumors were classified as ATM-activity low if the combined score was negative and as ATM-activity high if the combined score was positive. These classifications were applied to $\log _{2}$ expression values obtained with RMA on the metadata set described above.

Over-representation analysis. Over-representation analysis was performed with Gene Set Enrichment Analysis (GSEA) and Fisher's exact test. GSEA was used to investigate whether low-NDT-signature expression was associated with elevated activity of ATM. GSEA software (http://www.broadinstitute.org/gsea/index.jsp; ref. 64) was applied on $\log _{2}$ expression data of tumors classified as NDT-signature high or as NDT-signature low. GSEA returned the ATM-activity signature as upregulated in phenotype NDT-signature low (enrichment score $(\mathrm{ES})<0$ ) and significantly enriched at FDR $<25 \%$ when with 10,000 permutations of gene sets. To assess the overrepresentation of patients classified as NDT-signature high/ATM-activity low and of patients classified as NDT-signature low/ATMactivity high, we applied one-sided Fisher's exact test coded in $R$ to the contingency table obtained by classifying the metadata set with NDT and ATM-activity signatures. The Fisher's test indicated that tumors with low NDT-signature expression are significantly enriched in samples with high ATM-activity signature, and vice versa $(P<0.05)$.
Survival analysis. To evaluate the prognostic value of the NDT and ATM-activity signatures, we estimated the probability that patients would remain free of death (survival) with the Kaplan-Meier method. To confirm these findings, the Kaplan-Meier curves were compared with the log-rank test. $P$ values were calculated according to the standard normal asymptotic distribution. Survival analysis and Kaplan-Meier plots were obtained with the $R$ survcomp package. Kaplan-Meier curves have been compared with the log-rank test of the coin $R$ package.

Statistical analysis. Error bars are s.e.m., as indicated in the figure legends. $P$ values were calculated by Student's two-tailed $t$ test. For IHC, KruskalWallis chi-squared test and Spearman's rho test were used for the analysis, as indicated.

48. Brenner, S. The genetics of Caenorhabditis elegans. Genetics 77, 71-94 (1974).

49. Francis, R., Barton, M.K., Kimble, J. \& Schedl, T. gld-1, a tumor suppressor gene required for oocyte development in Caenorhabditis elegans. Genetics 139, 579-606 (1995).

50. Lee, M.H. \& Schedl, T. Identification of in vivo mRNA targets of GLD-1, a maxi-KH motif containing protein required for $C$. elegans germ cell development. Genes Dev. $15,2408-2420$ (2001)

51. Ciofani, M. et al. Obligatory role for cooperative signaling by pre-TCR and Notch during thymocyte differentiation. J. Immunol. 172, 5230-5239 (2004).

52. Carpenter, A.E. et al. CellProfiler: image analysis software for identifying and quantifying cell phenotypes. Genome Biol. 7, R100 (2006).

53. Fitzgerald, D.J. et al. Protein complex expression by using multigene baculoviral vectors. Nat. Methods 3, 1021-1032 (2006).

54. Hashimoto, Y. \& Costanzo, V. Studying DNA replication fork stability in Xenopus egg extract. Methods Mol. Biol. 745, 437-445 (2011).

55. Francia, S. et al. Site-specific DICER and DROSHA RNA products control the DNA-damage response. Nature 488, 231-235 (2012).

56. Robertson, K., Hensey, C. \& Gautier, J. Isolation and characterization of Xenopus ATM (X-ATM): expression, localization, and complex formation during oogenesis and early development. Oncogene 18, 7070-7079 (1999).

57. Irizarry, R.A. et al. Exploration, normalization, and summaries of high density oligonucleotide array probe level data. Biostatistics 4, 249-264 (2003).

58. Tusher, V.G., Tibshirani, R. \& Chu, G. Significance analysis of microarrays applied to the ionizing radiation response. Proc. Natl. Acad. Sci. USA 98, 5116-5121 (2001).

59. Cordenonsi, M. et al. The Hippo transducer TAZ confers cancer stem cell-related traits on breast cancer cells. Cell 147, 759-772 (2011).

60. Fallarino, F. et al. Metabotropic glutamate receptor-4 modulates adaptive immunity and restrains neuroinflammation. Nat. Med. 16, 897-902 (2010).

61. Johnson, W.E., Li, C. \& Rabinovic, A. Adjusting batch effects in microarray expression data using empirical Bayes methods. Biostatistics 8, 118-127 (2007).

62. Rustighi, A. et al. Prolyl-isomerase Pin1 controls normal and cancer stem cells of the breast. EMBO Mol. Med. 6, 99-119 (2014).

63. Adorno, M. et al. A mutant-p53/Smad complex opposes p63 to empower TGFß-induced metastasis. Cell 137, 87-98 (2009).

64. Subramanian, A. et al. Gene set enrichment analysis: a knowledge-based approach for interpreting genome-wide expression profiles. Proc. Natl. Acad. Sci. USA 102, 15545-15550 (2005) 